\title{
Relaxation and Music Reduce Pain Following Intestinal Surgery
}

\author{
Marion Good, ${ }^{1 *}$ Gene Cranston Anderson, ${ }^{1 \dagger}$ Sukhee Ahn, ${ }^{1 \dagger}$ Xiaomei Cong, ${ }^{1 \S}$ \\ Michael Stanton-Hicks ${ }^{2 \|}$
}

\author{
${ }^{1}$ Frances Payne Bolton School of Nursing, Case Western Reserve University, 10900 Euclid Avenue, \\ Cleveland, OH 44106-4904 \\ ${ }^{2}$ Division of Anesthesiology, Pain Management and Research, The Cleveland Clinic Foundation, 9500 \\ Euclid Avenue, Cleveland, OH, 44106 \\ Accepted 22 January 2005
}

\begin{abstract}
Three nonpharmacological nursing interventions, relaxation, chosen music, and their combination, were tested for pain relief following intestinal (INT) surgery in a randomized clinical trial. The 167 patients were randomly assigned to one of three intervention groups or control and were tested during ambulation and rest on postoperative days 1 and 2. Pain sensation and distress were measured with visual analog scales (VAS). Multivariate analysis of covariance showed significantly less post-test pain in the intervention groups than in the control group on both days after rest and at three of six ambulation post-tests $(p=.024-.001)$, resulting in $16-40 \%$ less pain. Mixed effects after ambulation were due to the large variation in pain and difficulty relaxing while returning to bed; but post hoc explorations showed effects for those with high and low pain. These interventions are recommended along with analgesics for greater postoperative relief without additional side effects.@ 2005 Wiley Periodicals, Inc. Res Nurs Health 28:240-251, 2005
\end{abstract}

Keywords: postoperative; pain; music; relaxation; intestinal; surgery; minimization

Postoperative pain is intensely uncomfortable and can exacerbate the stress response, interfere with appetite and sleep, and contribute to complications and prolonged hospitalization (Acute Pain Management Guideline Panel, 1992). In a descriptive study of pain after intestinal (INT) surgery, pain sensation and distress were moderate to severe during the first 2 days, even with patient controlled analgesia (PCA), and one-third of the participants had pain-related sleep disturbances (Good, Stanton-Hicks et al., 2001). Pain was associated with delayed postoperative recovery and more complications. Nonpharmacological nursing interventions have been recommended as adjuvants to analgesics for reducing postoperative pain, but their use has not been reported in INT surgery patients. The objective of this study, therefore, was to examine the effects of

Contract grant sponsor: National Institute of Nursing Research, NIH (to M.G.);

Contract grant number: RO1 NR3933 (1994-2005).

* Professor.

'Edward J. and Louise Mellen Professor of Nursing.

Research Associate.

${ }^{\S}$ Project Director.

"Vice Chairman, Pain Management and Research.

Published online in Wiley InterScience (www.interscience.wiley.com)

DOI: 10.1002/nur.20076 
relaxation, music, and the combination of both on pain after INT surgery. In addition, intervention effects on heart and respiratory rates and postoperative recovery outcomes (sleep, recovery rate, and complications) were explored.

In a previous report of a randomized clinical trial of 500 abdominal surgery (ABD) patients, these same interventions reduced sensory and affective pain on postoperative days 1 and 2 at both ambulation and rest (Good et al., 1999 ${ }^{\mathrm{a}}$ ). The sample for the primary study included gynecological (GYN), INT, and urological surgery patients with abdominal incisions. The present study completes a series of four reports of secondary analyses from the primary intervention study, distinguished by superscript codes $\left({ }^{\mathrm{a}, \mathrm{b}, \mathrm{c}}\right)$ on the references and citations. In two secondary analyses, the investigators reported sub-samples of 80 control group gynecological surgery (GYN) patients (Good, Stanton-Hicks et al., 2000) and 38 control group INT surgery patients (Good, Stanton-Hicks et al., 2001). The third secondary analysis concerned the randomized clinical intervention trial of relaxation, music, and their combination in 311 GYN patients (Good, Anderson, Stanton-Hicks, Grass, \& Makii, 2002 ${ }^{\mathrm{c}}$ ). The sample in the INT intervention trial published here $(N=167)$ overlaps with the primary ABD intervention study sample and the INT control sample but neither of the GYN samples.

No research on relaxation and music interventions for pain in INT surgery patients was found. Previous investigators have generally reported that these interventions were effective, both individually and in combination, after abdominal cardiac, and GYN surgeries (Good, 1995; Good et al., 1999 ${ }^{\mathrm{a}}$; Good, Stanton-Hicks et al., 2000 ${ }^{\mathrm{b}}$; Locsin, 1981; Mullooly, Levin, \& Feldman, 1988; Nilsson, Rawal, Unestahl, Zetterberg, \& Unosson, 2001; Updyke, 1990; Voss \& Good, 2004). Relaxation techniques, such as deep breathing and jaw relaxation, have reduced postoperative pain after abdominal surgery (Flaherty \& Fitzpatrick, 1978; Good et al., 1999 ; Levin, Malloy, \& Hyman, 1987; Roykulcharoen \& Good, 2004; Wells, 1982) and orthopaedic surgery (Osterbrink $\&$ Evers, 2000). Music resulted in fairly large effect sizes in early clinical studies of children and adults with various types of pain: orthopaedic, obstetric, cardiac, gynecologic, dental, and terminal illness (Standley, 1986; Standley \& Hanser, 1995).

Because there are gender, anatomical, and surgical differences between patients undergoing GYN and INT surgery, this report is of the participants (male and female) who underwent procedures on the INT tract or its accessory organs, rather than females who underwent surgery on the reproductive organs in GYN studies. Food is digested and absorbed in the INT tract, making its recovery vital for energy and repair after surgery, whereas the female GYN organs are for reproduction. In addition, the INT surgeries took nearly an hour longer than GYN surgeries and most involved longer incisions spanning the upper and lower abdomen rather than just lower; patients were somewhat younger, and more had chronic pain.

Good and Moore (1996) proposed in their prescriptive pain management theory that nonpharmacological methods are necessary along with analgesic medication to reduce the sensory and affective components of acute pain. The mechanism of this effect is explained by the gate control theory: the perception of pain is decreased by gates, which are diverse points in the central nervous system where the transmission of noxious impulses to the cortex is repeatedly modulated, filtered, and abstracted by physical, cognitive, and emotional factors (Fuster \& Alexander, 1973; Melzack \& Wall, 1965; Willis, 1985). The general neurobiological centers for sensory pain are the sensory cortex and thalamus; the centers for affective pain are the anterior cingular cortex and the limbic system (Rainville, Bushnell, \& Duncan, 2000). Pain also produces cognitive arousal and wide ranging cortical activation involving attention, evaluation, and activities to decrease the pain (Price, 1999).

Patients report that they use relaxation and music to relax and distract themselves from pain (Good, Stanton-Hicks et al., 2000); this diminishes muscle and mental tension (Jacobson, 1938; Standley, 1986). Selective attention alerts the prefrontal cortex to the sound (e.g., music or relaxation instructions) rather than to the noxious input, and thereby inhibits pain (Fuster \& Alexander, 1973; Willis, 1985). Reduced muscular and mental tension also decrease sympathetic stimulation of the hypothalamus (Beary \& Benson, 1974). These processes inhibit transmission of afferent noxious impulses in the central nervous system by activating endogenous opiates, descending nerve impulses, and neuropeptides in the brain and spinal cord (Andy, 1983; Yezierski, Gerhart, Schrock, \& Willis, 1983). Recent imaging studies showed that distraction decreased reported sensory pain and also modulated activity in the sensory cortex, while verbal suggestions decreased reported affective pain and also modulated activity in the anterior cingular cortex (Rainville, Carrier, Hofbauer, Bushnell, \& Duncan, 1999). 
Music has additional benefits that relaxation exercises lack; it provides complex sound that includes characteristics such as pitch, rhythm, and tempo that evoke meaning and memories (McCaffrey \& Good, 2000). Different musical characteristics are processed in separate but overlapping neuronal matrices in both hemispheres of the brain (Altenmuller, 2001). Imaging studies have revealed activity in the ascending auditory pathway, the auditory cortex, the limbic system, and the motor centers (in response to music) (Griffiths, 1999). Directing attention toward auditory stimuli decreased reported sensory and affective pain (Carrier, Rainville, Paus, Duncan, \& Bushnell, 1998; Miron, Duncan, \& Bushnell, 1989), and brain scans have shown that a distracting cognitive task decreased regional cerebral blood flow in the sensory cortex (Bushnell et al., 1999; Petrovic, Petersson, Ghatan, Stone-Elander, \& Ingvar, 2000).

The following hypotheses were tested with a priori contrasts in postoperative patients following INT surgery:

1. Patients in the three treatment groups (relaxation, music, and combination) will have significantly less pain than those in the control group.

2. Patients who receive the combination of relaxation and music will have significantly less pain than those who receive single treatments (relaxation or music).

3. Patients who receive relaxation will have significantly less pain than those who receive music.

\section{METHOD}

\section{Sample}

The 167 patients who underwent INT surgery and who had completed at least one of the research tests were selected for this secondary analysis from the larger randomized clinical trial of 500 abdominal surgical patients (Good et al., 1999 ${ }^{\mathrm{a}}$ ). Patients were from two medical centers and two community hospitals in a large midwestern city. Patients eligible for this analysis were 2070 years of age, $M=43 \pm 13$, had abdominal incisions, used PCA, and ambulated after surgery. Patients with rectal, laparoscopic, or small incisions were excluded, as were those with epidural analgesia or opioid dependence. There was no significant difference in the size of each group: relaxation (26\%), music (29\%), combination (22\%), and control $(23 \%), \chi^{2}(3$, $N=167)=2.17, p=.54$. Half were tested at ambulation first $(n=86,51 \%)$ and half at rest first $(n=81,49 \%)$.

Convenience sampling was used in the original study. Research nurses met eligible participants after their preadmission testing appointment for consent, and they interviewed them for demographic and minimized variables. A computerized minimization program was then used to randomly assign participants to a control group or one of three experimental groups (relaxation, music, or the combination). Minimization provided between-group stratification on gender, surgical specialty, chronic pain, prior surgery, and antidepressant or benzodiazepine use.

Two hundred seventeen patients underwent INT surgery, and $50(23 \%)$ were lost to follow-up because they either were no longer eligible after surgery $(18 \%)$ or withdrew before postoperative tests $(5 \%)$. No significant differences in demographic variables were found between these and the 167 reported here. Eighty-six of these 167 $(52 \%)$ missed at least one of the four tests either because of withdrawal or refusal/inability to participate in a test. Those who missed tests did not differ by treatment group, gender, race, religion, smoking, or chronic pain from the 81 who completed all tests. Those who completed at least one of the four tests $(n=167)$ were included in the intention-to-treat analysis (Everitt \& Dunn, 1991).

Most participants were Caucasian (95\%), female $(60 \%)$, married (63\%), Protestant $(52 \%)$, employed (62\%), from households with a monthly income of $\$ 3,000$ or less $(58 \%)$, not taking steroids $(70 \%)$, or benzodiazepines or antidepressants (84\%) and had completed at least some college (62\%). Most did not drink alcohol daily $(81 \%)$, but $53 \%$ were smokers and had smoked $M=27 \pm 22$ pack-years (packs per day times number of years). Nearly half $(40 \%)$ had chronic pain that had lasted more than a month. Almost all (99\%) had undergone previous surgery, $M=4 \pm 4.5$ surgical procedures. Mean body mass index was $27 \pm .8$. Using 100 -mm visual analog scales (VAS), their worst past pain was severe, sensation, $M=81 \pm 19 \mathrm{~mm}$, and distress, $M=69 \pm 30 \mathrm{~mm}$.

Participants had abdominal surgery for mucous ulcerative colitis (28\%), Crohn's disease (28\%), colon cancer $(10 \%)$, diverticulosis $(6 \%)$, rectal cancer $(5 \%)$, adhesions (4\%), or other INT conditions $(19 \%)$. The average time spent in surgery was 3 hours, 40 minutes $(S D=1$ hour, 19 minutes). Participants received PCA initially and 
progressed to oral medication several days later. Most surgical incisions spanned both the lower and upper abdomen $(67 \%)$, with $27 \%$ only in the lower, and $6 \%$ only in the upper abdomen. Most $(87 \%)$ were vertical incisions with $7 \%$ horizontal and $5 \%$ oblique. Incisions were a mean of $19 \pm$ $7 \mathrm{~cm}$ in length. Type and length of surgery, and incision location, length, and direction were not correlated with post-test pain and were similar among the groups.

\section{Experimental Interventions}

Preoperative teaching. With an introductory tape, participants assigned with the jaw relaxation technique were taught to let the lower jaw drop slightly; keep the tongue quiet and resting in the bottom of the mouth; let the lips get soft; breathe slowly with a three-rhythm pattern of inhale, exhale, and rest; stop forming words; and do not even think words (Flaherty \& Fitzpatrick, 1978; Good, 1995). The sedative music was 60-80 beats per minute with a sustained melodic quality, controlled volume, and without lyrics, strong rhythms or percussion (Gaston, 1951). The music tapes are described in Good, Picot et al. (2000). Selections are identified in Good (1992), and examples can be found in Lai and Good (2005). All participants received instructions on getting out of bed and splinting their incisions.

The introductory music tape consisted of a halfminute excerpt of five types of soothing music, and participants chose one of these types (Good et al., 1999). The combination tape contained music and jaw relaxation instructions. In the music and combination groups, 27 (32\%) chose classical orchestral music, 22 (26\%) chose piano, 17 (20\%) chose slow jazz, $10(12 \%)$ chose synthesizer, and $9(11 \%)$ chose harp. The control group received the same research procedures, measures, and 10 minutes of conversation in place of the teaching tape

Postoperative intervention. Sixty-minute intervention tapes were used postoperatively with lightweight foam earphones and a small tape recorder. The 1-minute relaxation technique was repeated at 1-minute intervals throughout the tape. The music tape consisted of a number of selections of the chosen type of music, played continuously. The combination tape included the relaxation technique repeated occasionally with a background of the chosen music selections. The research nurse accompanied all participants during ambulation. The intervention groups listened to a tape, and the control group did not. During rest, the intervention groups listened to the tape, and the control group rested quietly for 15 minutes.

Mastery. Mastery, the ability to use the technique, was verified by observing for four criteria: (a) face relaxed, (b) no grimace or frown, (c) not talking, and (d) slow respirations. These observations were made during preoperative practice, at four points during postoperative ambulation, and before and after testing at rest. Mastery was defined as meeting three of the four criteria (2 points each). Mastery scores were highest before surgery with $92 \%$ of participants achieving mastery. Scores were $7.67 \pm .73$ points (of 8) during preoperative practice at rest; and $7.63 \pm .83$ points during practice while walking. Postoperatively, mastery scores were lower than preoperatively, with $78-93 \%$ achieving mastery at rest and fewer, 66-94\% achieving mastery during ambulation. Mastery improved significantly from pre-rest to post-rest on both days respectively, $t(122)=4.17, p<.001$ and $t(112)=3.22, p=.002$ on day 2 , but decreased significantly from preparatory to post-ambulation on both days, $t(83)=-4.86, p=.001$, and $t(101)=-3.78, p<.001$. Mastery scores did not differ by intervention group.

\section{Measures}

Sensation and distress of pain. Pain is an unpleasant sensory and affective experience associated with tissue damage, such as surgery. The sensory component, which is the physical feeling of hurt at the incision, was measured with the VAS sensation of pain scale. The affective component, which is the emotional distress associated with the sensation, was measured with the VAS distress of pain scale. Participants were asked to report sensation and distress of pain as the intensity of how they felt now, on dual $100 \mathrm{~mm}$ VAS scales at six times each day.

The VAS scales were adapted from Johnson (1973) numerical rating scales. The anchors were none to most sensation or most distress. Construct validity of the original scales was supported by Johnson, whose laboratory studies showed that subjects could differentiate between sensation and distress during induced ischemic pain. Concurrent validity was good when comparing the original scales to the Melzack (1975) McGill Pain Questionnaires Pain Rating ScaleRanked, with positive correlations for sensation $(r=.44, p<.001)$, and distress of pain $(r=.55$, $p<.001$; Good, 1995). In the primary study $(N=500)$, correlations of the Johnson scales with 
the VAS were very good, $r=.89-.92$ (Good et al., 2001).

Clinical measures. Opioid analgesics taken at the time of the tests were assessed by subtracting the milligrams shown on the PCA display before and after the test and converted to milligrams of morphine equivalent. Radial pulse and observed respiratory rates were obtained as pre- and posttest measures of sympathetic nervous system response. Each morning, participants reported whether they had slept well, fitfully (intermittently), or not at all (Melzack, 1975), scored dichotomously as good sleep $=2$ or fitful or no sleep $=1$. To assess recovery, the research nurse recorded the number of days until bowel sounds were heard, the nasogastric tube was removed, clear liquids were ordered, PCA was discontinued, and patient discharge, and also the number of those with complications in the first 2 days. The research nurses interviewed for chronic pain ( $>1$ month) by asking whether and how long there was pain before surgery. She asked whether they drank alcohol and the amount per day and whether they smoked, the number of cigarettes per day, and the number of years.

The medical chart was the source of data for the surgical procedure, postoperative diagnosis, length of surgery, recovery variables, and complications. Incision location and direction were observed when the length of incision was measured. During a final interview, those in the intervention groups were asked several structured questions: (a) whether they used the assigned intervention to relax, distract, or both (1-3 scale); (b) the amount the intervention helped their pain ( $0-3$ scale), rated as none, a little, a moderate amount, or a lot; and (c) whether the intervention reduced sensation, distress, or both. Those who received music were asked about (d) whether they liked it, (e) found it sedative, and (f) had increased feeling of control over pain (yes, no).

\section{Procedure}

Preoperative instruction. The investigational review board of each institution approved the study, and all participants gave written informed consent. In the preadmission clinic, participants were interviewed for demographic and minimization data and information on factors that might affect postoperative pain (e.g., chronic pain, smoking, and alcohol use). The research nurse taught the use of the pain sensation and distress scales and measured worst past pain. The research nurse then used the computerized minimization program to randomly assign participants to groups. Those in the treatment groups listened to the intervention teaching tapes and practiced the techniques.

Postoperative testing. At the bedside, testing at ambulation consisted of three phases: a 5minute preparatory period in bed; an ambulation period (getting out of bed, walking a comfortable distance, and returning to bed); and a 10-minute recovery period in bed. Those in the treatment groups listened to the tape throughout all three phases. The research nurse measured pain sensation and distress in all participants at four times during ambulation: before and after the 5-minute preparatory period, after ambulation, and after the 10-minute recovery from ambulation. Ninety percent of the testing was done between the hours of 9:30 AM and 3:45 PM; tests were separated by 1 hour to prevent carryover. Ambulation lasted $M=27 \pm 15 \mathrm{~min}$ on day 1 and $26 \pm 7$ min on day 2. Testing at rest consisted of measuring pain sensation and distress before and after the 15minute treatment tape or control condition of resting in bed.

The investigators used a standardized procedure to train research nurses. Interrater reliability was tested at intervals throughout the study, averaging $90 \%$ for vital signs, mastery, and chart extraction. To minimize equalization of treatments and resentful demoralization, all participants consented to receiving a "slight self-care addition to usual care," and were not told the other interventions. To prevent diffusion of treatments, those in different groups were not assigned to the same room, and staff was not told the content of the tapes. The tapes provided treatment consistency. Final interviews were done following all treatments.

\section{RESULTS}

\section{Pretest Equivalence}

Mean sensation and distress scores for each group at each of the four pretests (pre-prep and pre-rest, days 1and 2) and eight post-tests (post-prep, amb, recovery, and post-rest, days 1 and 2) are shown in Table 1 and Figure 1. Most pretest means on day 1 were $>40 \mathrm{~mm}$ for sensation $(75 \%)$ and $>30 \mathrm{~mm}$ for distress (100\%); on day 2, they were $>30 \mathrm{~mm}$ for both sensation (75\%) and distress (63\%). Large standard deviations $(17-32 \mathrm{~mm})$ indicated wide variation in pain (Table 1). There were no significant pretest group differences in sensation and distress. 


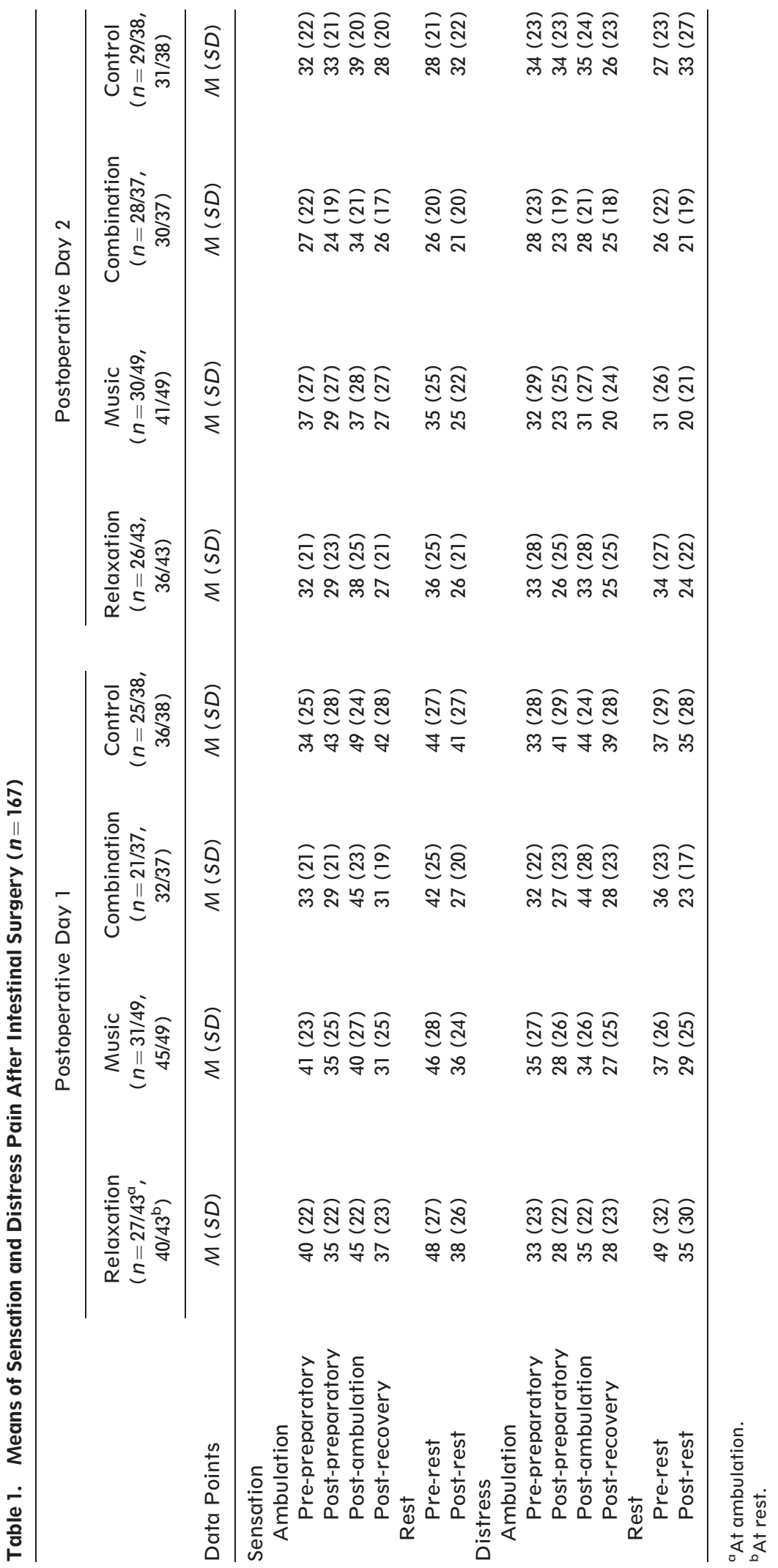


A Day 1 Sensation

$\rightarrow$-Relax $\rightarrow$-Music $\quad-\Delta$ Combination $\rightarrow$ Control

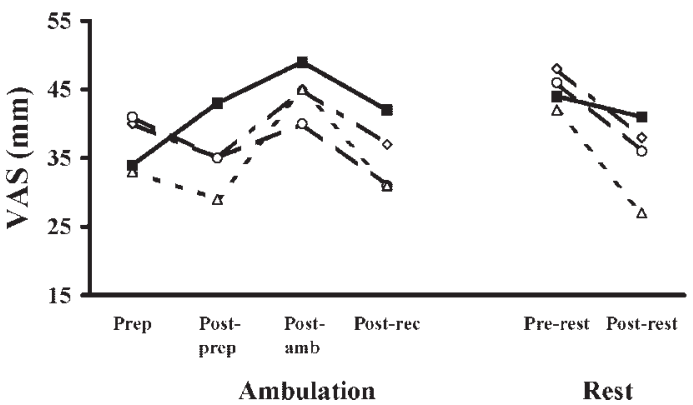

C

Day 2 Sensation

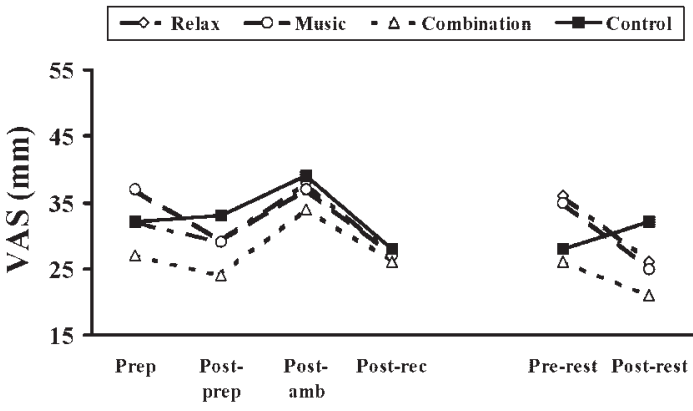

Ambulation

Rest
B

Day 1 Distress

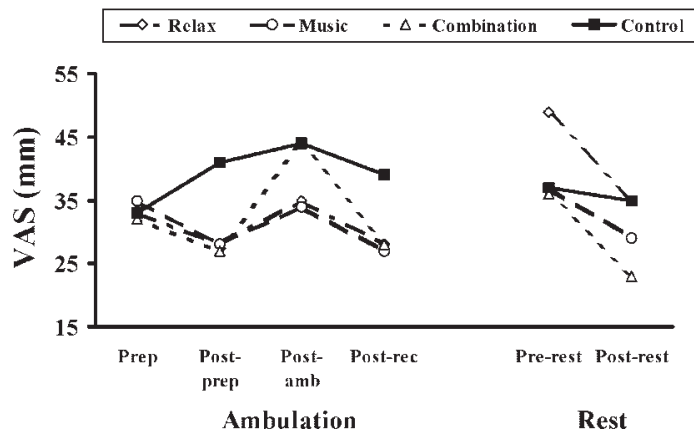

D

Day 2 Distress

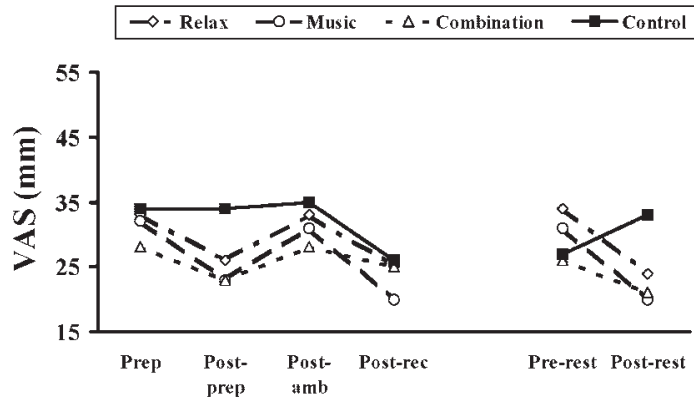

Ambulation

Rest

FIGURE 1. Mean sensation and distress of pain VAS scores after intestinal surgery are presented for postoperative days 1 and 2 at ambulation and at rest. Pretests at ambulation were preparatory (prep) measures of sensation and distress; three post-tests were post preparatory (post-prep) after $5 \mathrm{~min}$ preparation for ambulation; postambulation (post-amb) after ambulating and returning to bed; and post-recovery (post-rec), after 10 minutes recovery from ambulation. During rest, the pretest was pre-rest, and the post-test after 15 minutes of rest in bed was the post-rest.

None of the descriptive measures were correlated with post test pain sensation or distress at any of the tests: age, gender, education, marital status, race, religion, income, smoking, alcohol use, chronic pain, previous surgeries, preoperative pain, intensity of past sensation and distress, previous use of relaxation, or belief that the intervention would relieve pain. In addition, clinical events after randomization were not correlated with post-test pain scores: length of surgery, diagnosis of cancer, location and length of incision, PCA mg of morphine equivalent used during each test, or participants with PCA or other opioids medications at testing. Pretest sensation and distress scores were highly correlated with post-tests at each point, $r=.55-.86, p<.01$, and therefore were used as covariates (Cook \& Campbell, 1979).

\section{Multivariate Contrasts}

The orthogonal a priori contrasts were tested (onetailed) at the eight post-test data points (three during ambulation and one at rest each day) using MANCOVA, while controlling for pretest sensation and distress. The multivariate factor was posttest pain sensation and distress.

The three treatment groups taken together had significantly less post-test pain than the control group on day 1 at the post-preparatory, postrecovery, and post-rest measurement points, but not at post-ambulation (Table 2). On day 2, the treatment groups had significantly less post-test pain at the post-preparatory and post-rest points. Though results were not significant at postambulation on either day or at post-recovery on 
Table 2. MANCOVA Contrast 1: Treatments Versus Control After Intestinal Surgery $(n=167)$

\begin{tabular}{|c|c|c|c|c|c|c|c|c|}
\hline & \multicolumn{4}{|c|}{ Day 1} & \multicolumn{4}{|c|}{ Day 2} \\
\hline & $F$ & $d f$ & $p$ & Power & $F$ & $d f$ & $p$ & Power \\
\hline \multicolumn{9}{|l|}{ Ambulation } \\
\hline Post-preparatory & 8.66 & 2,97 & .001 & .96 & 4.01 & 2,106 & .011 & .69 \\
\hline Post-ambulation & 1.45 & 2,97 & ns & .30 & .24 & 2,106 & ns & .09 \\
\hline Post-recovery & 3.15 & 2,97 & .024 & .59 & .01 & 2,106 & ns & .05 \\
\hline \multicolumn{9}{|l|}{ Rest } \\
\hline Post-rest & 4.92 & 2,146 & .005 & .80 & 14.39 & 2,131 & .001 & 1.00 \\
\hline
\end{tabular}

Note: Only Contrast 1 is shown. It compares the three treatment groups together to the control group. Contrasts 2 and 3 were not supported. The multivariate dependent variable, pain, was composed of post-test sensation and distress; pretest sensation and distress were controlled as covariates.

day 2, differences between means were in the expected direction (Table 1). Thus there was mixed support for Hypothesis 1. There was no support for Hypothesis 2: the combination intervention group did not experience significantly less pain than the individual intervention groups. There was no support for Hypothesis 3: there was no significant difference in pain between the relaxation group and the music group. The interventions relieved pain at the majority of data points but they were similar in their effects.

\section{Exploratory Analyses}

Highs and lows. To examine reasons for the nonsignificant differences in pain after ambulation, exploratory subgroup analyses were done with pretest sensation scores divided at the 75th centile into highs $(n=28)$ and lows $(n=85)$. On day 1, treatment group differences were not significant for either highs or lows at the postambulation point when mastery scores were lowest. However on day 2, MANOVA indicated significant treatment effects at post-ambulation for 28 highs, $M=66 \pm 12 \mathrm{~mm}, F(2,23)=5.47$, $p=.006$, and 85 lows, $M=21 \pm 14 \mathrm{~mm}, F(2$, $79)=4.46, p=.02$; and at post-recovery for highs, $F(2, \quad 23)=2.68, \quad p=.045, \quad$ and lows, $F(2$, $80)=2.42, p=.048$. As the level of significance was not adjusted, these exploratory analyses must be interpreted cautiously.

Effect on recovery, heart, and respiration rates. In the treatment groups, pain sensation and distress were not related to sleep, recovery variables, or complications at any of the 12 data points; chi square analysis indicated that the treatment and control groups did not differ in these variables. In addition, MANOVA showed that there was no significant difference between the treatment groups and the controls on post-test pulse/respiratory rates.

\section{Additional Findings}

Positive patient reports at the end of the study supported the findings for Hypothesis 1 . In the treatment groups, $96 \%$ reported that the interventions were helpful for pain, and the majority (64\%) said they reduced pain a moderate amount or a lot and increased their feeling of being in control of pain $(62 \%)$. They reported that the interventions reduced sensation (7\%), distress $(47 \%)$, and both sensation and distress (38\%).

Participants used the music in different ways: $27 \%$ reported that they used the music to relax, $21 \%$ to distract themselves from the pain, and 52\% for both. Nearly all in the music and combination groups liked their chosen music a moderate amount to a lot $(96 \%)$, and $71 \%$ reported that the music was sedative. This was supported by reports that $70 \%$ were asleep at the end of one or more of the tests. Three who disliked the music but did not withdraw were included in the intent-to-treat analysis. In addition, most of those in the jaw relaxation group (83\%) used the technique independently without the tape.

Inquiry into their musical background revealed that most in the music and combination groups $(73 \%)$ were frequent music listeners, and half $(50 \%)$ were music performers either past or present. Most (86\%) reported that they would use the intervention again if they were having surgery, and $94 \%$ would recommend it to others.

Clinical significance. Effect sizes (ES) were calculated with post-test adjusted means $\left(d=\mathrm{m}_{1}-\mathrm{m}_{2} / \mathrm{s} d_{\mathrm{p}} ; f=d\right) ;$ small $\geq .10$, medium $\geq .25$, large $\geq .40$. In addition, percent less sensation and distress were used to describe clinical effects (Table 3). Comparing the three 
Table 3. Clinical Significance: Adjusted Differences and Percent Less Pain in Treatment Groups ( $n=167)$

\begin{tabular}{|c|c|c|c|c|c|c|c|c|}
\hline \multirow[b]{3}{*}{ Data Points } & \multicolumn{4}{|c|}{ Day 1} & \multicolumn{4}{|c|}{ Day 2} \\
\hline & \multicolumn{2}{|c|}{ Sensation } & \multicolumn{2}{|c|}{ Distress } & \multicolumn{2}{|c|}{ Sensation } & \multicolumn{2}{|c|}{ Distress } \\
\hline & Difference & $\%$ & Difference & $\%$ & Difference & $\%$ & Difference & $\%$ \\
\hline \multicolumn{9}{|l|}{ Ambulation } \\
\hline Post-preparatory & 12.81 & 28 & 13.71 & 33 & 5.41 & 16 & 7.10 & 22 \\
\hline Post-ambulation & 7.66 & 25 & 6.78 & 15 & 1.37 & $4^{a}$ & 2.27 & $7^{a}$ \\
\hline Post-recovery & 10.73 & 25 & 11.67 & 30 & .02 & $.1^{a}$ & .37 & $2^{a}$ \\
\hline \multicolumn{9}{|l|}{ Rest } \\
\hline Post-rest & 8.56 & 20 & 7.86 & 22 & 10.91 & 32 & 14.00 & 40 \\
\hline
\end{tabular}

Note: Contrast 1: Treatment-control group difference in post-tests after adjusting for pretests.

a Subsequent subgroup examination of the highs and lows separately on day 2 indicated greater relief than for the whole group. At post-ambulation, highs $(n=28)$ who used the interventions had $45 \%$ less sensation and $61 \%$ less distress than the controls, while the lows $(n=85)$ had $23 \%$ less sensation and $37 \%$ less distress. At post-recovery, the highs who used the interventions had $53 \%$ less sensation and $76 \%$ less distress while the lows had $24 \%$ less sensation and $38 \%$ less distress.

treatment groups to the control group, the ES for sensation and distress at significant data points were small to medium (.12-.31), but at nonsignificant points, there was no effect (.02-.16). Effects were large for the highs at post-ambulation on day $2(.47-.58)$; and were small to medium for the lows (.22-33). At post-recovery, ES were medium to large for the highs (.39-.43), and small to medium for the lows (.19-.27). Findings were supported by reports of the helpfulness of the interventions.

The percent less sensation and distress in the treatment groups than in the control group was calculated with adjusted scores. Differences ranged from 7 to $14 \mathrm{~mm}$ at significant points, or $16-40 \%$ less pain the intervention groups compared to using medication alone (Table 3).

\section{DISCUSSION}

In these INT surgery patients, relaxation, music, and the combination reduced pain at rest and at several ambulation points on postoperative days 1 and 2. Subgroup analyses of those with high and low pain on day 2 showed further effects at postambulation and post-recovery, which suggests that the initial lack of effect may be related to the large variance in pain scores. Thus the interventions provided many patients with clinically significant relief, which was supported by exit reports of helpfulness. The lack of significance at day 1 postambulation coincided with the lowest mastery score and may have been due to inadequate relaxation when getting back in bed. The interventions had no effect on any of the recovery variables or complications, and post-test pain in the treatment groups was not related to these outcomes. In the INT control group subsample in Good et al. (2001) $)^{\mathrm{b}}$, greater sensation and/or distress were significantly and moderately related to slower recovery at up to six data points. The brief interventions in the INT study seemed to eliminate relationships with recovery, but were not sufficient to actually improve recovery rates.

There was no effect on pulse or respiration rates; autonomic effects were not demonstrated following the INT surgeries, even though they were found at every data point in the primary ABD study and the GYN subsample (unpublished raw data, Good, Stanton-Hicks, Grass, \& Anderson, 1994-1998). The reason for this difference is not clear. In this smaller sample, the INT participants were younger (INT $M=43, \mathrm{ABD}=45$, $\mathrm{GYN}=46$ years) and more had chronic pain (INT $=40 \% ; \mathrm{ABD}=36 \% ; \mathrm{GYN}=33 \%$ ), perhaps associated with chronic stress and sustained sympathetic effects (McEwen, 2003; Walker \& Sofaer, 1998).

Methods of self-care use are an important part of relaxation and music for pain. The brief jaw relaxation technique provided relief and most participants continued to use the easily remembered sequence. Those with music were directed to use the music to relax and distract themselves from the pain, but only $52 \%$ used it both ways. This suggests that participants had developed their own ways of listening to music, which continued while using music for pain. Giving them directions cued them that they needed to participate. Use of music in the past may have influenced their own way of listening. The sedative effects of the music 
is consistent with sedative selections on the tapes, which also significantly improved sleep quality in Taiwanese elders (Lai \& Good, 2005).

The positive effects at rest are generally consistent with those of other studies of abdominal, orthopaedic, and GYN surgical patients who were not studied during ambulation (Levin et al., 1987; Locsin 1981; Mullooly et al., 1988; Nilsson et al., 2001; Osterbrink \& Evers, 2000; Updyke, 1990; Wells, 1982). In this INT group, significance was found at fewer ambulation points (3/6) than those in the primary $\mathrm{ABD}^{\mathrm{a}}$ study (6/6) or the GYN intervention study (6/6). Effects found in highs and lows at two more points suggest that lack of overall effects on day 2 was due to the small sample and large variance.

Evidence at most data points supported the Good and Moore (1996) theoretical proposition that nonpharmacological modalities (relaxation, music and their combination), in addition to analgesics, are helpful for satisfactory pain relief. In addition, there was exploratory support that relaxation and music are effective even when pain is severe or mild, but less effective when patients do not focus on them. These findings improve the predictive value of the theory. Patients used relaxation and music to relax and distract themselves from pain, which supports the gate control theory mechanisms that cognitive and emotional factors result in descending modulation of noxious impulses (Melzack \& Wall, 1965), mediated by the thalamus, the hypothalamus, autonomic nervous system, and endogenous opiates (Beary \& Benson, 1974; Dickenson, 1983; Hardy, 1985; Standley, 1986; Tasker, Choiniere, Libman, \& Melzack, 1987). Lack of autonomic effects suggests variations in the mediation.

It is recommended that researchers try longer listening times during the first 2 days to see if a larger dose improves pain, physiological measures, opioid intake, side effects, sleep, recovery, and complications. They could also compare pain after getting back in bed to pain at other points in the ambulation process: when they stand, walk, turn back, and reach the bedside.

The results can be generalized to populations of INT surgery patients that are similar to this one: middle-aged Caucasian males and females undergoing specific surgeries in urban and suburban hospitals in the US. Surgical procedures will differ in other hospitals and over time as surgical practice changes. Similarity of effects for the three interventions across the ABD, GYN, and INT intervention studies is probably due to the common mechanisms of relaxation and/or dis- traction. With similar effects, it is recommended that nurses give patients choices among these interventions, encourage sequential use, and suggest using the music to relax or distract from pain.

The five choices of music have been found to be acceptable and effective for most participants in African American, Asian, Egyptian, and Caucasian populations (Good et al., 2000; Lai \& Good, 2005; Phumdoung \& Good, 2003, Salem, 2004; Voss \& Good, 2004), but choices were broader when culturally relevant music was offered (Lai \& Good; Voss \& Good). At exit interviews after each study, participants have suggested other types of music including more culturally specific selections. Relaxation techniques can also be culturally related: a music/relaxation tape spoken by an African American voice is available (Campinha-Bacote, Campinha-Bacote, \& Allbright, 1992). Nurses who consider patient preferences can offer a variety including culturally relevant choices (Good, Picot et al., 2000).

In conclusion, relaxation, choice of music, and the combination of the two were effective in reducing postoperative sensation and distress of pain at ambulation and rest on day 1 and 2. They can be used in addition to analgesics, and when reminders to relax are given, they can provide patients with additional comfort without the additional side effects of opioid analgesics.

\section{REFERENCES}

(References marked with a superscript [a,b,c] indicate the few analyses discussed in text)

Acute Pain Management Guideline Panel. (1992). Acute pain management: Operative or medical procedures and trauma. Clinical practice guideline (AHCPR Publication No. 92-0032). Rockville, MD: Agency for Health Care Policy and Research, Public Health Service, US Department of Health and Human Services.

Altenmuller, E.O. (2001). How many music centers are in the brain? Annals of the New York Academy of Sciences, 930, 273-280.

Andy, O.J. (1983). Thalamic stimulation for chronic pain. Applied Neurophysiology, 46, 116-123.

Beary, J.F., \& Benson, H. (1974). A simple psychophysiologic technique which elicits the hypometabolic changes of the relaxation response. Psychosomatic Medicine, 36, 115-120.

Bushnell, M.C., Duncan, G.H., Hofbauer, R.K., Ha, B., Chen, J. I., \& Carrier, B. (1999). Pain perception: Is there a role for primary somatosensory cortex? Proceedings of the National Academy of Sciences of the United States of America, 96, 7705-7709. 
Campinha-Bacote, J., Campinha-Bacote, D., \& Allbright, J. (1992). C.A.R.E. (Culturally-specific Africentric Relaxation Exercise). Transcultural C.A.R.E. Associates, 11108 Huntwicke Place, Cincinnati, OH, 45241.

Carrier, B., Rainville, P., Paus, T., Duncan, G.H., \& Bushnell, M.C. (1998). Attentional modulation of pain-related activity in human cerebral cortex. Social Neuroscience Abstracts, 24, 1135.

Cook, T.D., \& Campbell, D.T. (1979). Quasiexperimentation: Design \& analysis issues for field settings. Boston: Houghton Miller.

Dickenson, A. (1983). The inhibitory effects of thalamic stimulation on the spinal transmission of nociceptive information in the rat. Pain, 17, 213-224.

Everitt, B.S., \& Dunn, G. (1991). Applied multivariate data analysis. New York: Halstead.

Flaherty, G.G., \& Fitzpatrick, J.J. (1978). Relaxation technique to increase comfort level of postoperative patients: A preliminary study. Nursing Research, 27, $352-355$.

Fuster, J.M., \& Alexander, G.E. (1973). Firing changes in cells of nucleus medialis dorsalis associated with delayed response behavior. Brain Research, 61, 79-91.

Gaston, E.T. (1951). Dynamic music factors in mood change. Music Educators Journal, 37, 42-44.

Good, M. (1992). Comparison of the effects of relaxation and music on postoperative pain. Unpublished dissertation, Cleveland $\mathrm{OH}$ : Case Western Reserve University.

Good, M. (1995). A comparison of the effects of jaw relaxation and music on postoperative pain. Nursing Research, 44, 52-57.

Good, M., Anderson, G. C., Stanton-Hicks, M., Grass, J.A., \& Makii, M. (2002). ${ }^{\text {c }}$ Relaxation and music reduce pain after gynecologic surgery. Pain Management Nursing, 3, 61-70 (the reference marked with superscript 'c' indicates the four secondary analyses discussed).

Good, M., \& Moore, S.M. (1996). Clinical practice guidelines as a new source of middle-range theory: Focus on acute pain. Nursing Outlook, 44, 74-79.

Good, M., Picot, B.L., Salem, S.G., Chin, C.C., Picot, S.F., \& Lane, D. (2000). Cultural differences in music chosen for pain relief. Journal of Holistic Nursing, 18, 245-260.

Good, M., Stanton-Hicks, M., Grass, J.M., \& Anderson, G.C. (1994-1998). Effects of relaxation and music on postoperative pain. (Grant Number RO1 NR3933). Bethesda, MD: National Institute of Nursing Research, National Institutes of Health.

Good, M., Stanton-Hicks, M., Grass, J.M., Anderson, G. C., Choi, C.C., Schoolmeesters, L., et al. (1999). ${ }^{\text {a }}$ Relief of postoperative pain with jaw relaxation, music, and their combination. Pain, 81, 163-172 (the reference marked with superscript ' $a$ ' indicates the four secondary analyses discussed).

Good, M., Stanton-Hicks, M., Grass, J.A., Anderson, G. C., Makii, M., \& Geras, J. (2000). ${ }^{\text {b Pain after }}$ gynecologic surgery. Pain Management Nursing, 1, 96-104 (the reference marked with superscript ' $b$ ' indicates the four secondary analyses discussed).

Good, M., Stanton-Hicks, M., Grass, J.A., Anderson, G. C., Salman, A., \& Duber, C. (2001). ${ }^{\text {b }}$ Pain outcomes after intestinal surgery. Outcomes Management for Nursing Practice, 5, 41-46 (the reference marked with superscript ' $b$ ' indicates the four secondary analyses discussed).

Good, M., Stiller, C., Zauszniewski, J.A., Anderson, G.C., Stanton-Hicks, M., \& Grass, J.A. (2001). Sensation and distress of pain scales: Reliability, validity and sensitivity. Journal of Nursing Measurement, 9, 219-238.

Griffiths, T.D. (1999). Human complex sound analysis. Clinical Science (London), 96, 231-234.

Hardy, S.G. (1985). Analgesia elicited by prefrontal stimulation. Brain Research, 339, 281-284.

Jacobson, E. (1938). Progressive relaxation. Chicago: University of Chicago Press.

Johnson, J.E. (1973). Effects of accurate expectations about sensations on the sensory and distress components of pain. Journal of Personality and Social Psychology, 27, 261-275.

Lai, H., \& Good, M. (2005). Music improves sleep quality in older adults. Journal of Advanced Nursing, 49(3), 234-244.

Levin, R.F., Malloy, G.B., \& Hyman, D.B. (1987). Nursing management of postoperative pain: Use of relaxation techniques with female cholecystectomy patients. Journal of Advanced Nursing, 12, 463-472.

Locsin, R.G. (1981). The effect of music on the pain of selected post-operative patients. Journal of Advanced Nursing, 6, 19-25.

McCaffrey, R.G., \& Good, M. (2000). The lived experience of listening to music while recovering from surgery. Journal of Holistic Nursing, 18, 378390.

McEwen, B.S. (2003). Mood disorders and allostatic load. Biological Psychiatry, 54, 200-207.

Melzack, R. (1975). The McGill Pain Questionnaire: Major properties and scoring methods. Pain, 1, 277299.

Melzack, R., \& Wall, P.D. (1965). Pain mechanisms: A new theory. Science, 150, 971-979.

Miron, D., Duncan, G.H., \& Bushnell, M.C. (1989). Effects of attention on the intensity and unpleasantness of thermal pain. Pain, 39, 345-352.

Mullooly, V.M., Levin, R.F., \& Feldman, H.R. (1988). Music for postoperative pain and anxiety. Journal of the New York State Nurses Association, 19, 4-7.

Nilsson, U., Rawal, N., Unestahl, L.E., Zetterberg, C., \& Unosson, M. (2001). Improved recovery after music and therapeutic suggestions during general anaesthesia: A double-blind randomised controlled trial. Acta Anaesthesiologica Scandinavica, 45, 812817.

Osterbrink, J., \& Evers, G.C. (2000). The influence of nursing measures on incision pain and the use of opioids in the postoperative period. Pflege, 13, 306314. 
Petrovic, P., Petersson, K. M., Ghatan, P.H., StoneElander, S., \& Ingvar, M. (2000). Pain-related cerebral activation is altered by a distracting cognitive task. Pain, 85, 19-30.

Phumdoung, S., \& Good, M. (2003). Music reduces sensation and distress of labor pain. Pain Management Nursing, 4, 54-61.

Price, D.D. (1999). Brain processing of pain and analgesia. In D.D. Price (Ed.), Physiological mechanisms of pain and analgesia: Vol. 15, Progress in pain research and management (pp. 97-135). Seattle: International Association for the Study of Pain.

Rainville, P., Bushnell, M.E., \& Duncan, G.H. (2000). PET studies of the subjective experience of pain. In K.L. Casey \& M.C. Bushnell (Eds.), Pain imaging (pp. 123-156). Seattle, WA: International Association for the Study of Pain.

Rainville, P. Carrier, B. Hofbauer, R.K., Bushnell, M.C., \& Duncan, G. H. (1999). Dissociation of sensory and affective dimensions of pain using hypnotic modulation. Pain, 82, 159-171.

Roykulcharoen, V., \& Good, M. (2004). Systematic relaxation to relieve postoperative pain. Journal of Advanced Nursing, 48, 140-148.

Salem, S. (2004). The effect of music on pain during the first stage of labor in Egypt. Unpublished doctoral dissertation, Cleveland, OH: Case Western Reserve University.
Standley, J.M. (1986). Music research in medical/dental treatment: Meta-analysis and clinical applications. Journal of Music Therapy, 23, 56-122.

Standley, J.M., \& Hanser, S.B. (1995). Music therapy research and applications in pediatric oncology treatment. Journal of Pediatric Oncology Nursing, 12, 3-8; discussion 9-10.

Tasker, R.A., Choiniere, M.,Libman, S.M., \& Melzack, R. (1987). Analgesia produced by injection of lidocaine into the lateral hypothalamus. Pain, 31, 237-248.

Updyke, P.A. (1990). Music therapy results for ICU patients. Dimensions in Critical Care Nursing, 9, 39-45.

Voss, J., \& Good, M. (2004). Sedative music reduces anxiety and pain during chair rest after open-heart surgery. Pain, 112, 197-203.

Walker, J., \& Sofaer, B. (1998). Predictors of psychological distress in chronic pain patients. Journal of Advanced Nursing, 27, 320-326.

Wells, N. (1982). The effect of relaxation on postoperative muscle tension and pain. Nursing Research, 31, 236-238.

Willis, W.D. (1985). The pain system. New York: Karger.

Yezierski, R.P., Gerhart, K.D., Schrock, B.J., \& Willis, W.D. (1983). A further examination of effects of cortical stimulation on primate spinothalmic tract cells. Journal of Neurophysiology, 49, 424-441. 